\title{
Especificidad de la Creatividad: Figurativa y Científica
}

\author{
Ana Bernal, Javier Esparza \\ María-José Ruiz, Mercedes Ferrando, Marta Sainz
}

Departamento Psicología Evolutiva y de la Educación, Universidad de Murcia, Murcia

\section{España}

Correspondencia: Mercedes Ferrando. Facultad de Educación, Campus de Espinardo, Murcia, C.P.30100. España. E-mail: mferran@um.es

(C) Universidad de Almería and Ilustre Coelgio Oficial de la Psicología de Andalucía Oriental (Spain) 


\section{Resumen}

Introducción. Este trabajo aborda la cuestión sobre la naturaleza de la creatividad como una habilidad general o específica de cada dominio concreto. Se exponen, en primer lugar, las principales definiciones y modelos del constructo; y en segundo lugar, el debate sobre la naturaleza generalista o específica de las habilidades creativas, tema que está siendo fuente de investigación para muchos autores.

Método. Han participado 133 estudiantes de $3^{\circ}$ de ESO pertenecientes a Instituto Público de un municipio de la Región de Murcia (España). Los instrumentos utilizados han sido: el test de creatividad figurativa de Torrance (TTCT), el Test de Pensamiento Científico-Creativo (TPCC), y la Batería de Aptitudes Diferenciales (DAT-5). La relación entre los constructos de creatividad figurativa-general y creatividad científica ha sido estudiada utilizando distintas técnicas estadísticas: correlaciones, análisis factorial y mapeado perceptual elaborado a través del escalamiento multidimensional.

Resultados. Los resultados nos indican la especificidad de ambos constructos, es más, dada las correlaciones entre la tarea "Manzanas" del TPCC y el juego 1 del TTCT, se podría hablar de una especificidad de tarea.

Discusión y conclusiones. El debate sobre la generalidad versus especificidad de la creatividad tiene una repercusión directa en la práctica educativa. Concretamente, esta repercusión se da en dos aspectos muy importantes: a) en el tipo de evaluación necesaria para identificar el potencial creativo, y por tanto, a los alumnos susceptibles de entrar en los programas específicos de atención a la diversidad de las altas habilidades; y b) en cuanto a la trascendencia en cómo enseñar y entrenar el pensamiento divergente y la creatividad de los estudiantes.

Palabras Clave: Especificidad de la Creatividad; Creatividad Científica; Test de Torrance; Pensamiento divergente 


\begin{abstract}
Introduction. This work tackles the question about the nature of creativity as a general vs. domain-specific ability. First we deal with the concept of creativity and then, the main approaches to the debate of generality vs. specificity are exposed

Method. A total of 133 students attending 3rd grade of secondary education in Murcia Region (Spain) took part in this research. The assessment instruments were the Torrance Test of Creative Thinking to assess general-figurative creativity and the $\mathrm{Hu}$ and Adey's ScientificCreative thinking test to meassure scienific creativity. Also the Diferencial Aptitude Test was used to meassure intelligence. The relationship between figurative-general creativity and scientific creativity was studied from diferent statistical procedures: using correlation analysis, factorial, and using perceptual mapping.
\end{abstract}

Results. The results point out to the independence between both figurative and scientific creativity; further more, we could talk about a task-specific creativity.

Discussion or Conclusion. The debate about the generality vs domain specific creativity does have a great effect on the educational practice. Specifically regarding two issuees: a) the type of assessment to identify the students' creative potential, thus deciding which students could be candidates to enter specific gifted and talented programs; b) regarding to how to teach and foster divergent thinking and creativity in our students.

Keywords: Specificity of creativity; Scientific creativity; Torrance test; Divergent thinking. 


\section{Introducción}

En los últimos años se está debatiendo ampliamente sobre si la creatividad es una habilidad general o específica de cada dominio (Plucker, 2004; Silvia, Kaufman \& Pretz, 2009). Aunque hay evidencias de que las personas muestran un rendimiento creativo sólo en algunas áreas, esta especificidad puede deberse a las características propias del dominio en cuestión y al conocimiento previo requerido, y no tanto a una especificidad de las habilidades creativas (Baer, 1998; 1999).

En el contexto escolar la importancia sobre la generalidad versus especificidad de la creatividad viene marcada por las implicaciones educativas que esto supone: si la creatividad es una habilidad general, entonces puede ser entrenada de forma diversa, esperando que los estudiantes transfieran las habilidades adquiridas a distintos ámbitos y dominios de su vida cotidiana y/o académica. Si, por el contrario, la creatividad supone una habilidad específica de dominio, las actividades generalistas de entrenamiento del pensamiento divergente no serían efectivas para fomentar la creatividad en ciencias o en música, por ejemplo. Es por ello, que este debate adquiere interés para los maestros y educadores que quieran fomentar un pensamiento creativo en sus estudiantes. Porque si la creatividad es de domino específico, dicho pensamiento no puede desligarse del campo o área en que se quiere fomentar. Este debate no afecta sólo al entrenamiento de la creatividad, sino también a la identificación de la misma. Así, la identificación del talento en áreas concretas no sería evaluable con pruebas generalistas, independientemente del dominio o área de interés. La identificación de la creatividad en un domino requerirá de pruebas específicas del mismo.

Autores como Sak y Ayas (2011) revisan los principales trabajos que abogan por la necesidad de utilizar medidas y modelos de contenido específico para evaluar la creatividad, sea cual sea el dominio (lengua, ciencias, arte, etc.). En este debate sobre la generalidad o especificidad de la creatividad encontramos tres posturas delimitadas. En la primera de ellas, tenemos autores que defienden la creatividad como dominio general; particularmente en los comienzos del estudio científico de la creatividad, autores como Guilford (1950) y Torrance (1962), defienden la creatividad como una habilidad general y transferible (Bermejo, Ruiz, Prieto, Ferrándiz, \& Sáinz, 2015); es decir, la persona creativa posee unas habilidades generales (independientes del dominio) y, por tanto, se pueden extrapolar de un dominio a otro. 
Gabora (2010) pone a prueba la hipótesis de que el estilo creativo de los individuos no se utiliza de forma exclusiva en un dominio concreto, sino que es transferible a otros dominios. Gabora defiende la generalidad de la creatividad basada en la existencia de habilidades comunes a los diferentes dominios, pero incidiendo en la necesidad de poseer conocimiento específico dentro de cada campo. En su estudio, participaron siete estudiantes de escritura creativa en la Universidad de Columbia Británica (Canadá) y se utilizaron dos instrumentos de evaluación diseñados ad-hoc: uno en el que se le pedía a los estudiantes que realizaran una obra de arte plástica; el otro estuvo basado en el autoinforme de percepciones para valorar tanto el trabajo propio como el de los otros compañeros. Los resultados probaron que la familiaridad que el individuo tenía con su trabajo creativo en un dominio concreto, facilita el reconocimiento del trabajo creativo de ese individuo tanto en su campo, como en otro dominio diferente.

La segunda postura sobre la creatividad como dominio específico ha ganado muchos seguidores en los últimos años (Baer, 2012; 2014; Han \& Marvin, 2002; Kaufman \& Baer, 2004; Reiter-Palmon, Illies, Kobe, Buboltz \& Nimps, 2009; Silvia et al., 2009; Tsai, 2014). Por ejemplo, Han y Marvin (2002) realizaron un trabajo en el que se estudió (en una muestra de 109 estudiantes de entre 7 y 8 años) la especificidad de la creatividad según el dominio y si ésta podía predecirse en base al pensamiento divergente (general). Utilizaron tres pruebas: a) las subpruebas de usos alternativos y similitudes del test de creatividad de Wallach-Kogan (1965) para medir la creatividad verbal; b) Real-World Divergent Thinking Test (Okuda, Runco, \& Berger, 1991) para medir la creatividad en la resolución de problemas de la vida escolar; y c) tres tareas de rendimiento en tres dominios diferentes (matemáticas, literatura y realización de collages). Los resultados obtenidos apoyaron la posición del dominio específico, ya que los niños exhibieron distintas capacidades creativas en diferentes dominios (matemáticas, literatura, y realización de collage), en lugar de una capacidad creativa uniforme en todos los dominios, lo que indicó que existe una considerable variación intra-individual en la capacidad creativa.

En el trabajo de Kaufman y Baer (2004) se aborda el tema desde el estudio de la autopercepción de la creatividad. Administraron dos escalas diseñadas ad hoc (Escala de Personalidad Creativa y un autoinforme para evaluar la creatividad en diferentes dominios) a 241 
estudiantes universitarios en distintos dominios académicos (biología, historia, psicología de la educación,...). Aunque los autores partían de una hipótesis que apoyaba la concepción de la creatividad como habilidad específica, sus resultados apoyan que ésta es percibida por los participantes como un constructo mucho más general. Los autores, pudieron diferenciar usando el analisis factorial tres grandes dominios de la creatividad: 1) relaciones interpersonales, 2) trabajos manuales, y 3) otro relacionado con las matemáticas y las ciencias. Además, se encontró que los participantes eran consistentes en sus autopercepciones: si se auto-percibían como creativos de forma general, tendían a puntuarse alto en todos los dominios, a excepción del dominio de matemáticas. Los autores explican estos resultados porque las matemáticas son vistas como un área que no exige expresar pensamientos creativos.

Tsai (2014) realiza un estudio exploratorio cuyos participantes fueron 17 escolares taiwaneses de 10 años de edad. Se emplearon tres medidas para el estudio del potencial creativo: un test de creatividad verbal (Guilford, 1967), un test de creatividad figurativa (Jellen \& Urban, 1986) y un auto-informe sobre el comportamiento creativo (Runco, Plucker \& Lim, 2001). En su trabajo, a través del estudio de correlaciones y del estudio de mapeado perceptual (elaborado a través del escalamiento multidimensional), se confirma la especificidad de la creatividad. Los resultados obtenidos muestran que las creatividades verbal y visual pueden ser entendidas como dos constructos diferentes, lo cual lleva al autor a aceptar la tendencia de las teorías que defienden la creatividad de dominio específico.

Baer (1998; 1999; 2012; 2014), quizá es el autor que más ha estudiado la especificidad de la creatividad en muchos de sus diferentes trabajos en esta área, principalmente aportando revisiones de investigaciones previas. Este autor plantea que la especificidad puede llegar a ser concretada de manera muy precisa, tanto que el autor habla de microdominios dentro del dominio específico de la misma; es decir, que dentro de un dominio específico de la creatividad (como puede ser el arte), podemos encontrar a su vez diferentes microdominios (danza clásica, historia del arte, etc.).

Por último, la tercera postura sostiene una posición híbrida, es decir, entiende la creatividad como habilidad tanto de dominio general como específico. Entre los autores que apoyan esta visión se encuentran Plucker y Beguetto (2004), quienes proponen que la creatividad tiene componentes tanto específicos como generales, pero que el nivel de esta especificidad 
depende del contexto social y del desarrollo de la persona, de cómo es la transición a la vida adulta, ya que la creatividad tiene que ver con la estimulación que ofrezca el contexto en el que nos desarrollamos; por ejemplo, un niño que crece en un entorno de músicos, será muy probablemente más propenso a desarrollarse de manera creativa en el campo de la música.

También encontramos el modelo jerárquico APT (Amusement Park Theoretical; Baer \& Kaufman, 2005), el cual partiendo de la "metáfora de un parque de atracciones" intenta explorar la creatividad. Los primeros requisitos son: inteligencia, creatividad y ambiente. Estos requisitos deben estar presentes en un cierto nivel durante todo el trabajo creativo. Según Baer y Kaufman (2005), las áreas temáticas generales tienen dominios específicos, y a su vez dentro de los dominios específicos, existen microdominios.

Según Silvia et al. (2009), estos modelos híbridos podrían ser aceptados desde las dos posiciones, pero es poco probable satisfacer a los seguidores de los modelos específicos más estrictos, ya que después de todo, las teorías híbridas defienden que algunos rasgos de la creatividad tienen influencia en todos los dominios, que es justo el punto de desacuerdo.

\section{Objetivos}

El objetivo general del presente estudio es verificar la generalidad o especificidad de la creatividad, estudiando la relación entre dos dominios del pensamiento divergente: el figurativo y el científico. Los objetivos específicos son: estudiar la relación entre las aptitudes intelectuales y los componentes de las pruebas de creatividad figurativa y científica; profundizando en los perfiles creativos de los alumnos dependiendo de su rendimiento académico.

\section{Método}

\section{Participantes}

En este estudio han participado un total de 133 alumnos de $3^{\circ}$ de la ESO (edad media 14.60 años; $d t=.72$ ), de los cuales 58 eran chicos y 75 eran chicas. Se trata de una muestra intelectualmente muy heterogénea, según lo indican sus puntuaciones en la prueba DAT-5 (Bennett et al., 2000). La muestra está compuesta tanto por alumnado de bajo rendimiento (programa de diversificación curricular, 23 alumnos), como por alumnado de habilidades me- 
dias. El nivel sociocultural del alumnado es medio-alto y el contexto en el que se encuentra inmerso el Instituto de Educación Secundaria (IES) es en el centro urbano del municipio, cuya economía se basa en el sector primario (agricultura y ganadería porcina) y en el sector servicios, ya que este último ocupa al mayor segmento de la población.

\section{Instrumentos}

El Test de Aptitudes Diferenciales DAT-5 (Bennett et al., 2000), evalúa 7 aptitudes básicas: Razonamiento Verbal, Razonamiento Numérico, Razonamiento Abstracto, Razonamiento Mecánico, Relaciones Espaciales, Ortografía, Rapidez y Exactitud Perceptiva. La prueba ha mostrado tener adecuadas propiedades psicométricas para la población española con coeficientes de consistencia interna entre .75 y .92, según indica el manual.

El Torrance Thinking Creative Test (TTCT, Torrance, 1974). Concretamente se ha utilizado la prueba figurativa (Forma A) de la adaptación realizada por Prieto, López y Ferrándiz (2003) y por Prieto, López, Ferrándiz y Bermejo (2003). Esta prueba se compone de tres subtest:: hacer un dibujo, completar una figura o dibujo y construir figuras o dibujos a partir de 30 pares de líneas paralelas. El test mide las cuatro grandes habilidades del pensamiento divergente: fluidez, flexibilidad, originalidad y elaboración. El tiempo de realización es de 10 minutos para cada tarea. La prueba ha mostrado adecuadas propiedades psicométricas con muestra española (Ferrando, Ferrándiz, Bermejo, Sánchez, Parra, \& Prieto, 2007).

Para evaluar la creatividad científica se utilizó el Test de Pensamiento Científico Creativo (TPCC) de Hu y Adey (2002). Se compone de siete tareas: 1 "Cristal" (el estudiante debe pensar en todos los usos científicos diferentes que le daría a un trozo de cristal); 2 "Planeta" (debe plantear preguntas de carácter científico que le gustaría investigar si viajara a otro planeta); 3 "Bicicleta" (mejoras para hacerla más interesante, útil y bonita); 4 "Gravedad" (describir qué pasaría en el mundo si no hubiera gravedad); 5 "Cuadrado" (dibujar distintas formas de dividir un cuadrado en cuatro partes iguales); 6 "Servilletas" (diseñar experimentos para demostrar cuál de dos servilletas es mejor); y por último, 7 "Manzanas" (diseñar y dibujar una máquina para recoger manzanas). Estas siete tareas evalúan tres dimensiones de la creatividad: fluidez (número de ideas), flexibilidad (distintas perspectivas utilizadas) y originalidad (infrecuencia estadística de las respuestas). La prueba ha sido estudiada con población española obteniendo adecuados índices de fiabilidad (.89). Se ha optado por no utilizar la 
prueba "Cuadrado", debido a la baja fiabilidad de la misma (Ruiz, Bermejo, Prieto, Ferrándiz, \& Almeida, 2013; Ruiz, Ferrando, Bermejo \& Prieto, 2015).

\section{Procedimiento}

Los datos que presentamos se enmarcan en el proyecto Atención a la Diversidad de los estudiantes de altas Habilidades: superdotados y talentos (Ref: EDU2014-53646-R). Para la recolección de datos de dicho proyecto se contactó con distintos colegios, así como con los los directores y orientadores de esos centros. Se obtuvieron los consentimientos informados tanto de padres como de alumnos, a quines se les informó sobre los objetivos de la investigación y la confidencialidad de sus resultados. La participación en esta investigación no conlleva ninguna consecuencia negativa para los participantes. En cuanto a la recolección de datos se realizó en un total de tres sesiones en horas lectivas cedidas por el centro.

Las pruebas fueron corregidas según las indicaciones de cada manual. Usualmente se recomienda que las pruebas de pensamiento divergente sean valoradas por más de un evaluador, aunque los tests de pensamiento divergente actuales no insisten en ello (Artola, Ancillo, Mosteiro \& Barraca, 2010).

\section{Análisis de Datos}

Las puntuaciones de las pruebas fueron codificadas utilizando para ello el paquete estadístico SPSS versión 20 (IBM, 2011). Se han realizado principalmente análisis correlacionales, y de reducción de dimensiones (análisis de componentes principales). Siguiendo la investigación realizada por Tsai (2014), se ha realizado un análisis multidimensional, a través de la técnica de mapeado perceptual, con el fin de observar cuál es la distancia (en términos de puntuación) entre las distintas variables de los dos test de creatividad utilizados en este estudio. También se han realizado análisis de comparación de medias para profundizar en los perfiles creativos de los alumnos dependiendo de su rendimiento académico (alumnos del programa de diversificación versus sus compañeros).

\section{Resultados}

\section{Análisis previos}


Previo al análisis de componentes principales se ha llevado a cabo un estudio sobre las correlaciones entre las variables del TTCT y las del TPCC. En el análisis de dichas correlaciones debemos fijarnos en las correlaciones intra-test (entre variables del mismo test) y las inter-test (entre variables de distintos test). Referente a las variables intra-test para la prueba de creatividad científica (TPCC) oscilan de $r=.07$ (entre la originalidad de la tarea Planeta y la originalidad de la tarea Manzanas) a $r=.96$ (entre la fluidez de la tarea Bicicleta y flexibilidad de la tarea Bicicleta). En total, se dan 121 interacciones, siendo el promedio de la correlación de .43. La mayoría de las correlaciones son estadísticamente significativas (salvo 9). Las mayores correlaciones se dan entre variables que pertenecen a la misma tarea, más que entre las variables que miden la misma dimensión del pensamiento divergente a través de diferentes tareas.

Las correlaciones intra-test para la creatividad figurativa (TTCT), supusieron 52 interacciones. La mayor correlación se encuentra entre la originalidad y la fluidez del subtest 3 ( $r$ $=.941 ; p<.001)$ y la menor entre la flexibilidad del subtest 2 y la elaboración del subtest 1 ( $r$ $=.014 ; p>.05)$. El promedio de las correlaciones fue de .215 . De ellas, la mayoría son estadísticamente significativas (67.3\%). Estas correlaciones no parecen seguir un patrón por tareas, ni tampoco por dimensiones del pensamiento divergente.

En cuanto a las relaciones inter-test, es decir, las correlaciones entre el TTCT y el TPCC, se encontraron un total de 171 interacciones, la correlación más baja estuvo entre la fluidez de la tarea Gravedad y la originalidad del subtest 1 del TTCT $(r=-.011 ; p=.903)$ y la más alta entre la flexibilidad de la tarea Planeta y la elaboración del subtest 3 del TTCT ( $r=$ $.470 ; p<.001)$. El promedio de correlación obtenido es de .021, de las cuales el $63.52 \%$ son estadísticamente significativas. Estas correlaciones se pueden analizar según las dimensiones del pensamiento divergente que miden las variables (fluidez, flexibilidad, originalidad y elaboración), o por los subtests de cada prueba. Por dimensiones, el promedio de correlación en la fluidez (12 interacciones) es de $r=.23$. Un $70 \%$ de las interacciones en la dimensión fluidez del test de TPCC y del TTCT eran estadísticamente significativas. En la dimensión flexibilidad, el promedio de correlación fue de $r=.24$, siendo el $80 \%$ estadísticamente significativas. Por último, para la dimensión originalidad, se obtuvo un índice de correlación promedio de $r=.11$, siendo el $11.11 \%$ estadísticamente significativas. Es de resaltar que para todos los 
subtests del TTCT la dimensión que más correlacionó con las variables del TPCC es la elaboración.

Las correlaciones según los subtests de cada prueba, mostraron que es el subtest 3 del TTCT "líneas paralelas" el que mayores correlaciones presentó con las tareas del TPCC, seguido del subtest 2 y finalmente del subtest 1 .

\section{Análisis Factorial Exploratorio de las Variables del TTCT y del TPCC}

Tras comprobar la factorialidad de los datos $\left[\mathrm{KMO}=.784 ; \chi^{2}=2464.661 ; \mathrm{gl}=351 ; p<\right.$ $.001]$, se ha utilizado un análisis de componentes principales, el cual arrojó una solución de 8 componentes con autovalor superior a 1 . Estos explicaban un total de $84.18 \%$ de la varianza (ver Tabla 1).

Tabla 1. Solución rotada del análisis de componentes principales

\begin{tabular}{|c|c|c|c|c|c|c|c|c|c|}
\hline \multirow[b]{3}{*}{$\begin{array}{c}\text { Auto } \\
\text { valores }\end{array}$} & & \multicolumn{8}{|c|}{ Componentes } \\
\hline & & I & II & III & IV & $\mathrm{V}$ & VI & VII & VIII \\
\hline & $\begin{array}{l}\% \text { varianza expli- } \\
\text { cada }\end{array}$ & 32.634 & 14.314 & 10.217 & 6.998 & 6.364 & 5.559 & 4.124 & 3.974 \\
\hline .964 & ttc_3_flui & .934 & & & & & & & \\
\hline .948 & ttct_3_orig & .930 & & & & & & & \\
\hline .936 & ttct_3_flex & .929 & & & & & & & \\
\hline .863 & ORIG.planeta & & .852 & & & & & & \\
\hline .881 & FLEXI.planeta & & .833 & & & & & & \\
\hline .869 & FLUI.planeta & & .823 & & & & & & \\
\hline .903 & FLEXI.cristal & & & .923 & & & & & \\
\hline .919 & ORIGI.cristal & & & .913 & & & & & \\
\hline .944 & FLUI.cristal & & & .910 & & & & & \\
\hline .940 & FLUI.bici & & & & .853 & & & & \\
\hline .916 & FLEX.bici & & & & .849 & & & & \\
\hline .903 & ORIGI.bici & & & & .807 & & & & \\
\hline .942 & FLEX.gravedadk & & & & & .900 & & & \\
\hline .929 & ORIG.gravedad & & & & & .884 & & & \\
\hline .915 & FLUI.gravedadc & & & & & .802 & & & \\
\hline .903 & ORIGIN.servilleta & & & & & & .892 & & \\
\hline .938 & FLUI.servilleta & & & & & & .889 & & \\
\hline .869 & FLEXI.servilleta & & & & & & .851 & & \\
\hline .733 & ttct_2_orig & & & & & & & .795 & \\
\hline .875 & ttc_2_fluid & & & & & & & .781 & \\
\hline .782 & ttc_2_flex & & & & & & & .708 & \\
\hline .683 & ttct_1_elab & & & & & & & & .660 \\
\hline .531 & Manz_Funciones & & & & & & & & .637 \\
\hline .786 & ttct_2_Elab & & & & & & & .471 & .607 \\
\hline .394 & ttct_1_Ori & & & & & & & & .571 \\
\hline .633 & Manz_Original & & & & .480 & & & & .521 \\
\hline .830 & ttct_3_elab & & .494 & & & & & & .500 \\
\hline
\end{tabular}

Método de extracción: Análisis de componentes principales.

Método de rotación: Normalización Varimax con Kaiser.

Se han eliminado las cargas inferiores a .4 
Como se observa en la Tabla 1, las variables han quedado agrupadas por tareas, siendo la más importante la tarea 3 "líneas paralelas" del TTCT, que ocupa el primer componente y explica un $32.63 \%$ de la varianza. El segundo componente queda delimitado por las variables de la tarea "Planeta" del TPCC, y explica un $14.31 \%$ de la varianza. El tercero queda delimitado por las variables de la tarea "Cristal" del TPCC. El cuarto por las variables de la tarea "Bicicleta" del TPCC. El quinto por "Gravedad" del TPCC; el sexto por "Servilletas" del TPCC; el séptimo por el subtest 2 del TTCT, y el octavo y último componente, explicando un $3.9 \%$ de la varianza, compuesto por las variables de la tarea "Manzanas" del TPCC y el subtest 1 del TTCT más las elaboraciones del TTCT 2 y 3. Por tanto, este octavo componente se forma con el Factor 3 del TTCT definido por Ferrando et al. (2007) más la prueba "Manzanas" del TPCC.

\section{Mapeado Perceptual a través del Análisis Multidimensional}

Con el fin de mostrar la relación entre ambos tests de creatividad de forma gráfica, y para facilitar la interpretación de los resultados, se ha utilizado el mapeado perceptual, una técnica de análisis multidimensional (PROXCAL) que permite visualizar distancias entre variables. Tsai (2014) lo llevó a cabo con un número reducido de variables, los expertos aconsejan utilizar de 8 a 20 variables (Green, 1975). Es por eso que en lugar de tomar las tareas (según nos indica el análisis factorial previo), hemos decidido tomar las variables de cada test, utilizando puntuaciones z, ya que las variables están puntuadas en distintas escalas. Ver Figura 1. 


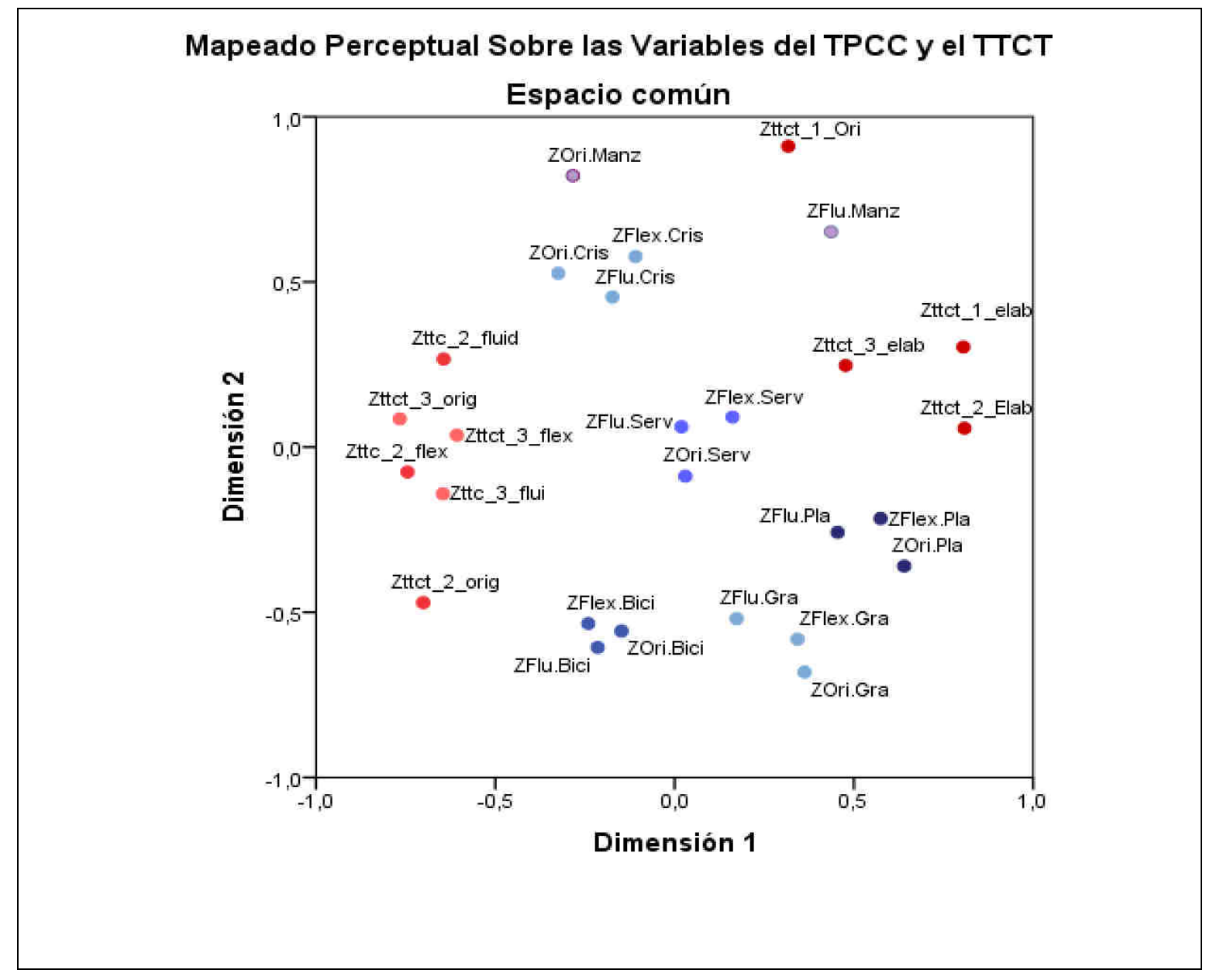

Figura 1. Mapa perceptual de las variables del TTCT y del TPCC

En la Figura 1 podemos observar la "situación" de las distintas variables de ambos test (TTCT y TPCC) en términos de distancia entre ellas. Es decir, a través del mapeado perceptual se nos indica la posición que cada variable adquiere con respecto a las demás. En el gráfico se han representado en el espectro de color rojo las variables del TTCT y en azul las del TPCC a fin de facilitar su visionado. Se observa que las variables se agrupan de acuerdo al componente principal en el que cargan dichas variables. Así, vemos que la distancia entre las variables del TTCT entre ellas mismas es menor que las de las variables del TPCC.

Las variables del Factor III del TTCT identificado por Ferrando et al. (2007) (originalidad del subtest 1 y elaboración de los tres subtests; en la esquina superior derecha del gráfico) se encuentran más alejadas del conjunto de variables del TTCT, además estas variables se ven más cercanas a las variables de la tarea "Manzanas" (en color lila). Como se puede apreciar en la constelación de puntos en color azul, las variables de las tareas "Cristal", "Planeta", 
"Bicicleta", "Servilleta" y "Gravedad" del TPCC se agrupan según la tarea a la que pertenecen. Estos resultados están en línea con los obtenidos en el análisis factorial exploratorio.

Relación con las Aptitudes Intelectuales medidas a través del DAT-5

Se ha estudiado la relación de las habilidades intelectuales con los componentes extraídos de las pruebas de creatividad figurativa y científica (Tabla 2). Teniendo en consideración los estudios previos sobre la estructura factorial de la prueba TTCT (Ferrando et al., 2007), se ha optado por agrupar las pruebas según resultados previos y utilizar el Factor I del TTCT (subtest 3 menos la elaboración), el Factor II (subtest 2 menos la elaboración) y el Factor III (elaboraciones de las tres tareas más la originalidad del subtest 1). Los componentes del TPCC corresponden con cada una de las tareas utilizadas. Ver Tabla 2.

Tabla 2. Matriz de correlaciones entre inteligencia (DAT) y creatividad

\begin{tabular}{lccccccc}
\hline & RV & RN & RA & RM & RE & Ort. & Rap. \\
\hline CRISTAL_total & .150 & .037 & -.059 & .052 & .139 & .076 & .188 \\
PLANETA_total & $.410^{* *}$ & $.448^{* *}$ & $.380^{* *}$ & $.294^{* *}$ & $.377^{* *}$ & $.393^{* *}$ & $.341^{* * *}$ \\
GRAVEDAD_total & $.235^{*}$ & $.351^{* *}$ & $.208^{*}$ & .138 & $.227^{*}$ & $.246^{*}$ & .119 \\
BICICLETA_total & .046 & .125 & -.073 & .049 & .019 & .071 & .071 \\
SERVILLETA_total & .193 & .156 & .036 & .062 & .104 & .170 & .208 \\
MANZANAS_total & .080 & .144 & .133 & $.201^{*}$ & .137 & .088 & .117 \\
FI:TTCT3_flu.flex.ori_total & -.010 & .055 & .014 & .031 & $.230^{*}$ & -.018 & .007 \\
FII:TTCT2_flu.flex.ori_total & .026 & .038 & -.074 & .043 & -.023 & -.019 & -.065 \\
FIII:TTCT1_.ori1.ela123_total & $.341^{* *}$ & $.356^{* *}$ & $.428^{* *}$ & $.365^{* *}$ & $.477^{* *}$ & $.368^{* *}$ & $.259^{*}$ \\
\hline
\end{tabular}

Nota: RV: Razonamiento Verbal, RN: Razonamiento Numérico; RA: Razonamiento Abstracto; RM: Razonamiento Mecánico; RE: Razonamiento Espacial, Ort.: Ortografía; Rap.: Rapidez Perceptiva. Todas las dimensiones fueron medidas utilizando la puntuación directa, no el percentil

(*) La correlación es significativa al nivel $\mathrm{p}<.05$.

(**) La correlación es significativa al nivel $\mathrm{P}<.001$.

El promedio de las correlaciones se situó en $r=.165$, mostrando correlaciones muy bajas entre ambos constructos que oscilaron entre $r=.007$ (Rapidez y Exactitud Perceptiva y el Factor I del TTCT) y $r=.477$ (entre Razonamiento Espacial y el Factor III del TTCTelaboraciones). Las tareas y los componentes que presentan mayores correlaciones con la inteligencia son la tarea "Planeta" y "Gravedad" del TPCC. Además, se observan las correlaciones más altas con el Factor III del TTCT. 
La aptitud intelectual que más correlaciona con el pensamiento creativo es el Razonamiento Espacial, que además de correlacionarse con las tareas "Planeta", "Gravedad" y el Factor III del TTCT muestra correlación con el subtest 3 del TTCT (líneas paralelas).

\section{Diferencias Según el Nivel de Rendimiento de los Alumnos}

Se ha estudiado si los alumnos del programa de diversificación curricular presentan rendimientos distintos en creatividad a sus compañeros. Debido a la naturaleza de las pruebas es de esperar que estas diferencias sean más acusadas en las tareas de creatividad científica, y no tanto en las tareas de creatividad figurativa medidas por el TTCT.

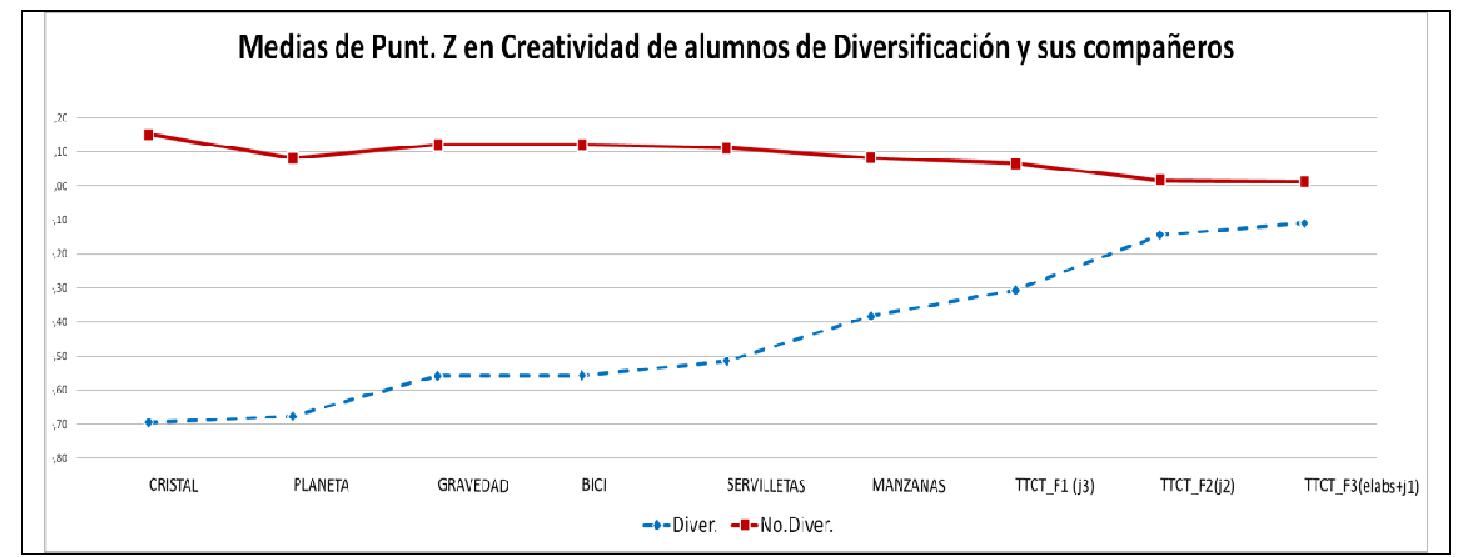

Figura 2. Gráfico de las puntuaciones medias en creatividad de los alumnos del programa de Diversificación y sus Compañeros (usando puntuaciones z).

Como muestran la Figura 2 y la Tabla 3, las puntuaciones medias del grupo de diversificación curricular, en todos los casos, son menores que las de sus compañeros, aunque se acercan mucho en el Factor II del TTCT y en el Factor I del TTCT. En cambio, las tareas en las que se ve más distancia son en "Planeta" del TPCC y en el Factor III del TTCT. A fin de facilitar la interpretación de dichas diferencias se han utilizado puntuaciones z para que todas las dimensiones aparezcan en la misma escala, tal como muestra la Figura 2. 
Tabla 3. Estadísticos descriptivos de las puntuaciones en creatividad de los alumnos del programa de diversificación y sus compañeros

\begin{tabular}{|c|c|c|c|c|c|c|c|}
\hline & \multicolumn{3}{|c|}{$\begin{array}{l}\text { Diversificación curri- } \\
\text { cular }\end{array}$} & \multicolumn{3}{|c|}{$\begin{array}{l}\text { No diversificación curricu- } \\
\text { lar }\end{array}$} & \multirow{2}{*}{ t de student } \\
\hline & $\mathrm{N}$ & M & dt. & $\mathrm{N}$ & M & dt. & \\
\hline CRISTAL_total & 23 & 5.00 & 5.25 & 106 & 10.72 & 9.49 & $\mathrm{t}(58,16)=-3.99 ; p<.001$ \\
\hline PLANETA_total & 23 & 19.17 & 11.11 & 106 & 33.45 & 16.88 & $\mathrm{t}(47,05)=-5.03 ; p<.001$ \\
\hline GRAVEDAD_total & 23 & 15.65 & 9.87 & 106 & 21.74 & 17.25 & $\mathrm{t}(55,71)=-2.29 ; p=.026$ \\
\hline BICICLETA_total & 23 & 14.57 & 8.62 & 106 & 24.28 & 14.73 & $\mathrm{t}(54,14)=-4.22 ; p<.001$ \\
\hline SERVILLETAS_total & 23 & 6.00 & 6.50 & 106 & 11.89 & 8.77 & $\mathrm{t}(127)=-3.03 ; p=.003$ \\
\hline MANZANAS_total & 23 & 8.48 & 6.32 & 106 & 11.12 & 5.46 & $\mathrm{t}(127)=-2.04 ; p=.043$ \\
\hline TTCT3_flu.flex.ori_total & 13 & 40.38 & 16.76 & 107 & 43.91 & 22.40 & $\mathrm{t}(118)=-.54 ; p=.585$ \\
\hline TTCT2_flu.flex.ori_total & 13 & 22.62 & 6.99 & 107 & 23.49 & 7.18 & $\mathrm{t}(118)=-.41 ; p=.680$ \\
\hline TTCT1_.ori1.ela123_total & 13 & 30.69 & 16.18 & 107 & 48.49 & 23.51 & $\mathrm{t}(118)=-2.64 ; p=.009$ \\
\hline
\end{tabular}

\section{Discusión y Conclusiones}

Quisieramos puntualizar que en esta investigación se han utilizado dos pruebas de pensamiento divergente (dominio figurativo y científico). El utilizar pruebas de pensamiento divergente en lugar de otro tipo de medidas tiene dos implicaciones: primera, como señala Plucker (2004), los resultados de las distintas investigaciones que han utilizado tests de pensamiento divergente tienden a apoyar la idea de una generalidad de la creatividad, mientras que los resultados de investigaciones basadas en pruebas de rendimiento en situaciones cotidianas (como escribir un poema, resolver un problema, etc.) tienden a apoyar la especificidad de dominio. Segunda, dado que la creatividad está afectada por la experiencia previa y el conocimiento base (Csikszentmihalyi, 1996; Sternberg \& Lubart, 1997), precisamente el utilizar pruebas de pensamiento divergente que, en nuestro caso, no requieren de un amplio conocimiento de dominio específico nos permite conocer si realmente las habilidades del pensamiento creativo son de dominio general o específico.

Los resultados de este estudio, procedentes de los distintos análisis nos trasladan a una misma idea: la relación intra-test entre las variables es mayor que la relación inter-test. Además, las distintas variables se relacionan más por las tareas a las que pertenecen que por las 
habilidades del pensamiento divergente que miden (fluidez, flexibilidad, originalidad y elaboración).

Nuestros resultados, vistos a priori, confirman la especificidad de la creatividad, estando en línea con los resultados hallados por Han y Marvin (2002), Kaufman y Baer (2004), Ferrándiz, Ferrando, Soto, Sainz y Prieto (2017); Ferrando, Esparza, Ruiz, Sainz, y Prieto (2017) y Tsai (2014). Incluso, los datos obtenidos en el análisis factorial apoyarían la idea de los microdominios defendida por Baer (2014), quien postula que el estudio de los dominios de la creatividad debe diseccionarse aún más. Recordemos que en nuestro estudio las variables se han agrupado por tareas específicas.

Sin embargo, el hecho de haber encontrado unas mayores correlaciones entre las variables de la prueba "Manzanas" del TPCC y el juego 1 del TTCT, nos lleva a cuestionarnos si esta especificidad se debe más a la naturaleza de las tareas que al dominio en sí mismo. Recordemos que los resultados del análisis factorial exploratorio y del mapeado perceptual arrojan unas soluciones de variables agrupadas según la naturaleza de la tarea y por tanto, por dimensiones del pensamiento divergente (fluidez, flexibilidad, originalidad y elaboración). En esta organización, la tarea "Manzanas" (TPCC) y el juego 1 (TTCT) parecen no discriminarse. Aunque hacen referencia a distintos dominios, en ambas tareas se solicita al alumno que realice un dibujo atendiendo a ciertas restricciones: una máquina para recoger manzanas (TPCC) o un dibujo utilizando un trocito de papel verde con forma de óvalo (TTCT). Estos resultados coinciden con los hallados por Diakidoy y Spanoudis (2002), quienes utilizaron dos pruebas distintas de pensamiento divergente: el TTCT verbal y una prueba paralela adhoc "Test de Creatividad en Historia", demostrando que la creatividad no sólo era específica de cada dominio sino, además, de cada tarea. Los autores aluden en sus conclusiones al posible efecto que la corrección y puntuación de las pruebas puede tener en estos resultados.

A la luz de estos resultados, podríamos plantearnos si en el caso de las investigaciones que se han basado en informes de autopercepción (Gabora, 2010; Kaufman \& Baer, 2004; Tsai, 2014), pudiera ser que los propios participantes asocien determinados tipos de tareas con ámbitos o dominios específicos. Pensamos que probablemente se asocia el dominio con el tipo de tareas que más lo define. Así, cabe pensar que el dominio de matemáticas, por ejemplo, esté implícitamente más asociado a la resolución de problemas planteados de forma explícita, en los que debe aplicarse algún tipo de algoritmo o fórmula para su resolución; mien- 
tras que el dominio de literatura, se asocia más a un fluir expresivo del pensamiento sin restricciones en la tarea.

Nuestros datos difieren del modelo que considera la creatividad de dominio general como una habilidad transferible entre dominios propuesto por los estudios pioneros de Guilford (1950) y Torrance (1974). Incluso, a la luz de nuestros resultados, se podría sostener que el conocimiento específico no es tan relevante a la hora de discriminar entre las habilidades del pensamiento divergente, como la forma de plantear dicho problema. Esto estaría en la línea de los trabajos de David Perkins (2003), quien defiende que el pensamiento creativo no se distingue del pensamiento ordinario; sino que lo que diferencia una solución creativa de una no creativa está en la naturaleza del problema planteado.

En cuanto a la relación entre ambos tipos de creatividad (figurativa y científica) y las aptitudes intelectuales, cabría esperar que dichas aptitudes correlacionaran más con la creatividad científica (TPCC) que con la figurativa (TTCT). Sin embargo, los resultados obtenidos muestran correlaciones muy bajas entre ambos tipos de creatividad y la inteligencia. Estos datos no están en la línea de otros estudios donde la relación entre el TTCT y la inteligencia fue de maginud media (Ferrando, Prieto, Ferrándiz \& Sánchez, 2005). En cuanto a los datos procedentes de la creatividad científica (TPCC) son dos las tareas que correlacionan de forma significativa con las aptitudes intelectules ("Planeta" y "Gravedad").

En resumen, los resultados sobre las correlaciones entre el constructo de la inteligencia y el de la creatividad (dominio figurativo y científico) no arrojan una respuesta clara sobre la diferenciación del pensamiento divergente por dominios, pues se vuelve a confirmar la especificidad por tareas más que por dominios.

En relación a las diferencias en creatividad dependiendo del rendimiento académico, los datos indican que los estudiantes del programa de diversificacion curricular puntúan significativamente por debajo del resto de sus compañeros. Estos datos concuerdan con los obtenidos por Ruiz (2013), quien estudió la relación entre creatividad científica (a través del TPCC) y el rendimiento académico, encontrando relaciones positivas y estadísticamente significativas a favor de los estudiantes con mayor rendimiento académico. Sin embargo, no se apreciaron diferencias estadísticamente significativas entre las puntuaciones del TTCT y el rendi- 
miento académico. Por ello, cabe plantearse si es más fácil ser creativo en tareas que conllevan la creación de dibujos (TTCT), sin requerir un conocimiento previo o en tareas más conectadas a un dominio (Ruiz, Bermejo, Ferrando, Prieto \& Sáinz, 2014).

Como apuntábamos al principio de este trabajo, el debate sobre la generalidad versus especificidad de la creatividad tiene una repercusión directa en la práctica educativa. Concretamente, como señalan Mohamed, Maker y Lubart (2012), esta repercusión se da en dos aspectos muy importantes: por un lado, en cuanto al tipo de evaluación necesario para identificar el potencial creativo y, por tanto, a los alumnos susceptibles de entrar en los programas específicos de atención a la diversidad de las altas habilidades. Además de la trascendencia sobre cómo enseñar y entrenar el pensamiento divergente de los estudiantes.

En nuestro estudio hemos utilizado pruebas de pensamiento divergente para medir el potencial creativo. Algunos expertos podrían aludir a que el pensamiento divergente no es "creatividad", y que la creatividad debería ser medida según los rendimientos reales y producciones novedosas aportadas por la persona a un campo concreto (Sternberg \& Lubart, 1997). Aún así, dado que las producciones reales están influenciadas por más factores que el pensamiento creativo por sí sólo (ej. formación, conocimientos previos, rasgos de personalidad e incluso suerte), hemos optado por utilizar pruebas de pensamiento divergente. La investigación futura en este campo debería ahondar en las diferencias entre distintos dominios, como el musical, para el que se disponen de pruebas específicas (Webster, 1983; Wang, 1985) o el pensamiento divergente del movimiento (Torrance, 1980). Además, es importante tener en cuenta la edad de los participantes. Sabemos que en edades tempranas la diferenciación entre distintas habilidades aún no es muy marcada, y el factor g tiene más relevancia que las habilidades específicas, mientras que en edades adultas esta diferenciación es mayor (Deary et al., 1996).

Otra línea de investigación interesante, es estudiar la transferencia en el entrenamiento del pensamiento divergente-creativo. Existen programas como el Mark diseñado por Renzulli (1973) que tratan el pensamiento creativo de una forma generalista; y otros programas destinados a adolescentes que se centran en un dominio específico, dentro de los cuales se trabaja el pensamiento creativo. Son ejemplos de estos programas los talleres de pensamiento científico para las altas habilidades de la Universidad de Murcia (cursos 2015-2016 y 2016-2017; 
Esparza, Ruiz, Bermejo, Ferrando \& Sainz, 2016), los cursos organizados desde el centro de superdotados de la Universidad de Anadolu (Sak, 2011); los talleres de Estimulación del Talento Matemático (ESTALMAT; Fernández Mota \& Pérez Jiménez, 2011) que se realizan en distintas comunidades autónomas; o el programa MENTORAC que se realiza desde la Universidad de Málaga (Fernández-Molina, Castro Zamudio, y Tomé Merchán, 2016). Saber si los niños que han recibido formación en un dominio específico de la creatividad pueden transferir y aplicar lo aprendido a otros dominios sería incluso más útil que centrarnos en la medición per se.

\section{Agradecimientos}

Este trabajo se ha realizado gracias a la ayuda recibida del Ministerio de Economía y Competitividad (Ref: EDU2014-53646-R).

\section{Referencias}

Artola, T., Ancillo, I., Mosteiro, P. y Barraca, J. (2010). PIC-N. Prueba de Imaginación Creativa para Niños. Madrid, España: Tea Ediciones.

Baer, J. \& Kaufman, J. (2005). Bridging generality and specificity: the Amusement Park Theoretical (APT) model of creativity (Theoretical and Interdisciplinary Perspectives). Roeper Review, 27, 158-163. DOI: http://dx.doi.org/10.1080/02783190509554310

Baer, J. (1998). The case for domain specificity in creativity. Creativity Research Journal, 11, 173-177. DOI: http://dx.doi.org/10.1207/s15326934crj1102_7.

Baer, J. (1999). Domains of creativity. En M. Runco \& S. Pritzker (Eds.), Encyclopedia of Creativity (pp. 591-596). New York: Academic Press.

Baer, J. (2012). Domain specificity of creativity: Theory, research and practise. Text: Creativity: Cognitive, Social and Cultural Perspectives. retrieved from http://www.textjournal.com.au/speciss/issue13/Baer.pdf

Baer, J. (2014). The crisis in creativity stems from too little fragmentation, not too much. Creativity, 1(2), 200-205. DOI: 10.15290/ctra.2014.01.02.04.

Bennett, G. K., Seashore, H. G. \& Wesman, A. G. (2000). DAT-5: Test de aptitudes diferenciales: versión 5. Manual. TEA Ediciones.

Bermejo, R., Ruiz, M. J., Prieto, M. D. Ferrándiz, C., \& Sáinz, M. (2015). Ensino do pensamento científico-criativo em estudantes do ensino médio. En Fátima Morais, Lúcia 
Miranda e Solange Weschler Creatividad: Aplicaciones prácticas en contextos internacionales (pp. 109-136). Brasil: Ediciones Vetor.

Csikszentmihalyi, M. (1996). Creativity. Flow and the Psychology of Discovery and Invention. New York: HarperCollins.

Deary, I. J., Egan, V., Gibson, G. J., Austin, E. J., Brand, C. R., \& Kellaghan, T. (1996). Intelligence and the differentiation hypothesis. Intelligence, 23(2), 105-132. DOI: https://doi.org/10.1016/S0160-2896(96)90008-2

Diakidoy, I. A. N., \& Spanoudis, G. (2002). Domain Specificity in Creativity Testing: A Comparison of Performance on a General Divergent Thinking Test and a Parallel, Content Specific Test. The Journal of Creative Behavior,36(1), 41-61. DOI: 10.1002/j.2162-6057.2002.tb01055.x.

Esparza, J., Ruiz, M.J., Bermejo, R., Ferrando, M. \& Sainz, M. (2016). Diseño de un programa para favorecer habilidades y estrategias del pensamiento científico-creativo. En F. Piske, T. Stoltz, S. Bahía y J. Machado (Eds.), Altas Habilidades /Superdotação (AH/SD) e Creatividade: Identificaçao e atendimento (pp 37-58). Brasil: Juruá Editora.

Feldhusen, J. F., Speedie, S. M. \& Treffinger, D. J. (1971). The Purdue Creative Thinking Program: Research and evaluation. NSPI Journal, 10(3), 5-9. DOI: $10.1002 /$ pfi.4180100304.

Fernández Mota, M.E. \& Pérez Jiménez, A. J (2011). Las Altas Capacidades y el Desarrollo del Talento Matemático. El Proyecto Estalmat-Andalucía. Revista Iberoamericana de Educación Matemática, 27, 89-113.

Fernández-Molina, M., Castro Zamudio, S., \& Tomé Merchán, D. (2016). Percepción y Grado de satisfacción familiar con el programa de Mentorías Universitarias para ACIs MENTORAC UMA 2013-2014. Talincrea. Talento, inteligencia y creatividad, 2(2), 18-31.

Ferrándiz, C., Ferrando, M., Soto, G., Sainz, M. \& Prieto, M. D. (2017). Divergent thinking and its dimensions: What we talk about and what we evaluate? Anales de Psicología, 33(1), 40-47. DOI: http://dx.doi.org/10.6018/analesps.32.3.224371.

Ferrando, M., Esparza, J., Ruiz, M.J., Sainz, M., \& Prieto, L. (2017). Especificidad del pensamiento divergente en música. En Fernanda Hellen Riveiro Psike, Carla Luciane Blum Vestena, Tania Stoltz, Jarci Maria Machado, Ana Aparecida de Oliveira Machado Bardy, Sara Bahia \& Samarah Perszel De Freitas (organizadores). Processsos Afe- 
tivos e Cognitivos de Superdotados e Talentosos (pp. 77-101). Curitiva (Brasil): Editora Prismas.

Ferrando, M., Ferrándiz, C., Bermejo, M.R., Sánchez, C., Parra, J. y Prieto, M. D. (2007). Estructura interna y baremación del Test de Pensamiento Creativo de Torrance. Psicothema, 19(3), 489-496.

Ferrando, M., Prieto, M.D., Ferrándiz, C. y Sánchez, C. (2005). Inteligencia y creatividad. Revista Electrónica de Investigación Psicoeducativa, 3(3), 21-50.

Gabora, L. (2010). Recognizability of creative style within and across domains: Preliminary studies. Proceedings of the Annual Meeting of the Cognitive Science Society. August. Portland, Oregon.retrived from http://arxiv.org/ftp/arxiv/papers/1005/1005.1518.pdf

Guilford, J. (1950). Creativity. American Psychologist, 5, 444-454.

Guilford, J. (1967). The nature of human intelligence. New York: McGraw-Hill.

Han, K.S. \& Marvin, C. (2002). Multiple Creativities? Investigating Domain - Specifity of Creativity in Young Children. Gifted Child Quarterly, 46(2), 98 - 109. DOI: https://doi.org/10.1177/001698620204600203.

Hu, W. \& Adey, P. (2002). A scientific creativity test for secondary school students. International Journal of Science Education, 24(4), 389-403. DOI: http://dx.doi.org/10.1080/09500690110098912.

IBM Corp. (2011). IBM SPSS Statistics for Windows, Version 20.0. Armonk, NY: IBM Corp.

Jellen, H.G., \& Urban, K.K. (1986). The TCT-DP (test for creative thinking - drawing production) an instrument that can be applied to most age and ability groups. The Creative Child and Adult Quarterly, 6(3), 138-155.

Kaufman, J.C. \& Baer, J. (2004). Sure, I'm creative - but not in mathematics!: self-reported creativity in diverse domains. Empirical studies of the Arts, 22(2), 143-155. DOI: https://doi.org/10.2190/26HQ-VHE8-GTLN-BJJM.

Mohamed, A., Maker, C.J. \& Lubart, T. (2012). Exploring the domain specificity of creativity in children: the relationship between a non-verbal creative production test and creative problem-solving activities. Turkish Journal of Giftedness and Education, 2(2), 84-101.

Okuda, S. M., Runco, M. A., \& Berger, D. E. (1991). Creativity and the finding and solving of real-world problems. Journal of Psychoeducational Assessment, 9, 45-53. DOI: https://doi.org/10.1177/073428299100900104. 
Perkins, D. (2003). La bañera de Arquímedes y otras historias del descubrimiento científico: el arte del pensamiento creativo. Barcelona: Editorial Paidós.

Plucker, J. A. (2004). Generalization of creativity across domains: Examination of the method effect hypothesis. Journal of Creative Behavior, 38, 1-12. DOI: 10.1002/j.21626057.2004.tb01228.x.

Plucker, J.A., \& Beghetto, R.A. (2004). Why creativity is domain general, why it looks domain specific, and why the distinction does not matter. En R. J. Sternberg, E.L. Grigorenko, \& J.L. Singer (Eds.), Creativity: From potential to realization (pp. 153-167). Washington, DC: American Psychological Association.

Prieto, M.D., López, O. y Ferrándiz, C. (2003). La creatividad en el contexto escolar. Estrategias para favorecerla. Madrid: Pirámide.

Prieto, M.D., López, O., Ferrándiz, C. y Bermejo, M.R. (2003). Adaptación de la prueba figurativa del Test de Pensamiento Creativo de Torrance en una muestra e los primeros niveles educativos. Revista de Investigación Educativa, 21, 201-213.

Reiter-Palmon, R., Illies, M. Y., Kobe, L., Buboltz, C. \& Nimps, T. (2009). Creativity and domain specificity: The effect of task type on multiple indices of creative problemsolving. Psychology Faculty Publications, 29, 2-30. DOI: http://dx.doi.org/10.1037/a0013410.

Renzulli, J.S. (1973). New Directions in Creativity . New York: Harper \& Row.

Ruiz, M. J. (2013). Estudio del Pensamiento Científico-Creativo en una muestra de alumnos de Educación Secundaria. Trabajo fin de Máster. Universidad de Murcia.

Ruiz, M. J., Ferrando, M., Bermejo, R., \& Prieto, M. D. (2015). Análisis Factorial Confirmatorio del test de Hu \& Adey: una medida de pensamiento científico-creativo. In I Jornadas de Doctorado. Universidad de Murcia. del 8 y 29 de abril de 2015. Retrieved from http://congresos.um.es/jdoctorado/jdoctorado2015/paper/viewFile/42111/17261

Ruiz, M. J., Bermejo, M. R., Ferrando, M., Prieto, M. D. \& Sáinz, M. (2014). Inteligencia y pensamiento Científico-Creativo: su convergencia en la explicación del rendimiento académico de los alumnos. Electronic Journal of Research in Educational Psychology, 12(2), 283-302. DOI: http://dx.doi.org/10.14204/ejrep.33.13122.

Ruiz, M. J., Bermejo, M.R., Prieto, M.D., Ferrándiz, C. y Almeida, L.S. (2013). Evaluación del pensamiento científico-creativo: Adaptación y validación de una prueba en población española. Revista Galego-Portuguesa de Psicoloxía e Educación, 21(1), 175-194. 
Runco, M.A, Plucker, J.A., \& Lim, W. (2001). Development and psychometric integrity of measure of ideational behaviour. Creativity Research Journal, 13(3\&4), 393-400. DOI: http://dx.doi.org/10.1207/S15326934CRJ1334_16.

Sak, U. \& Ayas, M.B. (2011). Creative Scientific Ability Test (C-SAT). Manuscrito sin publicar.

Sak, U. (2011). An overview and social validity of the education programs for talented students model (EPTS). Egitim ve Bilim, 36(161), 213-229.

Silvia, P.J., Kaufman, J.C. \& Pretz, J.E. (2009). Is Creativity Domain-Specific? Latent Class Models of Creative Accomplishments and Creative Self-Descriptions. Psychology of Aesthetics, Creativity, and the Arts, 3 (3), 139-148. DOI: http://dx.doi.org/10.1037/a0014940.

Sternberg, R.J. y Lubart, T.I. (1997). La creatividad en una cultura conformista. Un desafío a las masas. Barcelona: Paidós.

Torrance, E. P. (1974). The Torrance Tests of Creative Thinking - Technical Manual Research Edition - Verbal Tests, Forms A and B - Figural Tests, Forms A and B. Princeton NJ: Personnel Press.

Torrance, E.P. (1962). Guiding creative talent. Englewood Cliffs, N.J.: Prentice-Hall.

Torrance, E.P. (1980). Thinking creatively in action and movement. Athens, Georgia: Georgia Studies of Creative Behavior.

Tsai, K.C. (2014). Examining gender differences in creativity. International Journal of Social Sciences, 13(1), 115-122.

Wallach, M. A. \& Kogan, N. (1965). Modes of thinking in young children: A study of the creativity-intelligence distinction. New York: Holt, Rinehart and Winston.

Wang, C. (1985). Measures of Creativity in Sounds and Music (MCSM). Retrieved February 16, 2005, from http://www.uky.edu/cecilia/MCSM.

Webster, P. R. (1983). Refinement of a measure of musical imagination in young children and a comparison to aspects of musical aptitude. Paper presented at the Loyola Symposium on Creativity, New Orleans. 